\title{
Clinical outcomes and associated factors of radioiodine-131 treatment in differentiated thyroid cancer with cervical lymph node metastasis
}

\author{
CHUNG-JIE CAO ${ }^{1}$, CHENG-YUN DOU ${ }^{2}$, JIAYAN LIAN ${ }^{1}$, ZHAO-SHENG LUAN $^{3}$, \\ WEN ZHOU ${ }^{3}$, WENLIN XIE $^{1}$, $\mathrm{LI} \mathrm{CHEN}^{4}, \mathrm{KEHUA} \mathrm{ZHOU}^{4}$ and $\mathrm{HONG} \mathrm{LAI}^{4}$ \\ ${ }^{1}$ Department of Pathology, The First Affiliated Hospital of Sun Yat-Sen University, Guangzhou, \\ Guangdong 510080; ${ }^{2}$ Department of Hepatology, Qilu Hospital of Shandong University, Jinan, \\ Shandong 250012; ${ }^{3}$ Department of Nuclear Medicine, PLA 88 Hospital, Taian, Shandong 271000; \\ ${ }^{4}$ Department of Endocrinology, Qilu Hospital of Shandong University, Jinan, Shandong 250012, P.R. China
}

Received December 25, 2016; Accepted November 29, 2017

DOI: $10.3892 / \mathrm{ol} .2018 .8270$

\begin{abstract}
Cervical lymph node metastasis (CLNM) is common in differentiated thyroid cancer (DTC). Radioiodine-131 $\left({ }^{131} \mathrm{I}\right)$ treatment is recommended for the removal of residual thyroid tissue following thyroidectomy. To date, the effect of ${ }^{131} \mathrm{I}$ therapy on the outcomes of patients with DTC with CLNM is unclear. The aim of the present study was to evaluate the final outcome of patients with DTC with CLNM according to ${ }^{131} \mathrm{I}$ administration, and to analyze the factors that may affect clinical outcomes. A total of 357 patients with DTC with CLNM were recruited and divided into three groups: Those who received 2, 3 or 4 doses of ${ }^{131}$ I therapy, respectively. Successful ablation was defined as levels of stimulated serum thyroglobulin $<2 \mathrm{ng} / \mathrm{ml}$ in the absence of CLNM. The rates of successful ablation were 80.35 (229/285), $76.36(42 / 55)$ and $70.59 \%$ (12/17) for patients who received 2, 3 and 4 doses, respectively. The patients with DTC with CLNM who were $<45$ years old, with tumor sizes $<2 \mathrm{~cm}$, solitary nodules and TNM stage I-II disease exhibited significantly higher rates of successful ablation compared with the patients who were $\geq 45$ years old, with tumor size $\geq 2 \mathrm{~cm}$, multiple nodules and stage III-IV disease. Multivariate analyses revealed that tumor size, number of nodules and TNM stage were independent risk
\end{abstract}

Correspondence to: Dr Hong Lai, Department of Endocrinology, Qilu Hospital of Shandong University, 107 Wenhuaxi Road, Jinan, Shandong 250012, P.R. China

E-mail: laihong185@126.com

Abbreviations: DTC, differentiated thyroid cancer; CLNM, cervical lymph node metastasis; ${ }^{131}$ I, radioiodine-131; WBIS, whole-body iodine scan; Tg, thyroglobulin; Anti-Tg, anti-thyroglobulin antibodies; RSS, recurrence risk stratification

Key words: differentiated thyroid cancer, cervical lymph node metastasis, radioiodine-131, successful ablation factors associated with successful ablation in patients with DTC with CLNM who received 2 doses of ${ }^{131} \mathrm{I}$ therapy. ${ }^{131} \mathrm{I}$ administration is a useful therapy to eradicate cervical lymph node metastasis in patients with DTC, and may be preferentially indicated in patients with DTC with CLNM who are aged $<45$ years, with tumor sizes $<2 \mathrm{~cm}$, solitary nodules and lower TNM stages, in order to control and prevent recurrence and/or metastases.

\section{Introduction}

Thyroid cancer is the most frequent thyroid malignancy, accounting for $90 \%$ of all types of endocrine malignant cancer, and its incidence has increased markedly worldwide within the last few decades (1). Differentiated thyroid cancer (DTC) is the most common subtype and has a relatively good prognosis, with 10-year survival rates of 92-98\% (2). Unlike the majority of malignancies, cervical lymph node metastasis (CLNM) is identified in 53\% patients with DTC at initial surgery (3) and may be present even when the primary tumor is contained within the thyroid (4). CLNM is considered a risk factor for poor clinical outcome (5). Increased mortality rates and decreased survival have been demonstrated among patients with DTC with lymph node metastasis (6).

Total or subtotal thyroidectomy followed by radioiodine-131 $\left({ }^{131} \mathrm{I}\right)$ therapy and lifelong thyroid hormone suppressive therapy has been accepted as essential management for DTC (7). The first sufficient dose of ${ }^{131} \mathrm{I}$ administration following thyroidectomy to eradicate the remnant normal thyroid tissue is termed 'remnant ablation' (8). The goal of remnant ablation is to achieve an undetectable serum thyroglobulin $(\mathrm{Tg})$ level, which serves as a tumor marker to facilitate follow-up of biochemical analysis, to eradicate any neoplastic foci to decrease the risk of recurrence and to detect any recurrence by ${ }^{131}$ I scanning (9). Total thyroidectomy, together with central compartment dissection was recommended for patients with DTC (10), and if the pathological results verified the presence of lymph node metastasis, the first follow-up whole-body iodine scan (WBIS) was performed 6 months subsequent to the thyroidectomy to 
confirm the presence or absence of any CLNM. The patients exhibiting CLNM were then scheduled for a second ablative dose of ${ }^{131} \mathrm{I}(11)$.

Although ${ }^{131}$ I has been used for over 6 decades, the majority of previously published studies focused on a single optimal dose of remnant ablation (12). No data in the literature concentrated on the effect of repetitive ${ }^{131} \mathrm{I}$ administration in the treatment of DTC with CLNM. The aim of the present study was to evaluate whether ${ }^{131} \mathrm{I}$ administration has an effect on the final outcome of patients with DTC with CLNM and to analyze the associated factors responsible for the clinical outcomes.

\section{Materials and methods}

Patients. A total of 562 patients with DTC who received ${ }^{131}$ I administration following subtotal or total thyroidectomy were recruited from the Department of Endocrinology, Qilu Hospital of Shandong University (Shandong, China), from January 2012 to April 2016. All patients were classified according to the American Joint Committee on Cancer/Union for International Cancer Control 2010 risk stratification system (13), and the American Thyroid Association staging system was designed to assess the risk of recurrence in DTC (14). All patients had undergone thyroidectomy, together with neck lymphadenectomy and without ${ }^{131} \mathrm{I}$ treatment prior to enrolment. Histopathology revealed the diagnosis of papillary or follicular thyroid carcinoma with known positive CLNM at the time of primary surgery. The tissues were fixed in 10\% neutral buffered formalin (Sakura Finetek USA, Inc., Torrance, CA, USA) for $20 \mathrm{~h}$, overnight, embedded in paraffin at $56^{\circ} \mathrm{C}$ and sectioned at $4 \mu \mathrm{m}$ for hematoxylin (cat no. C0107; Biyuntian Biotech Co., Ltd., Shanghai, China.) and eosin (cat no. C0109; Biyuntian Biotech Co., Ltd.) staining (H\&E staining). Tissue sections were treated with hematoxylin stock solution (no dilution) for $30 \mathrm{~min}$ at room temperature, washed with double distilled water and followed immediately immersed in eosin stock solution (no dilution) for $30 \mathrm{~min}$. Images of H\&E-stained sections were captured using a x20 objective magnification attached to a Olympus DP71 digital camera Olympus microscope equipped with a Olympus DP71 digital camera (Olympus Life Science Imaging Systems Inc., Markham, ON, Canada). The diagnosis was performed by three veterinary pathologists. Pregnant woman and patients with distant metastases, white blood cell counts $<3.0 \times 10^{9}$ cells $/ 1$ (normal range, $4.0 \times 10^{9}-10.0 \times 10^{9}$ cells $/ 1$ ), severe hepatic and renal failure, poor wound healing and a recent history of iodine contamination were excluded. The Medical Ethical Committee of Qilu Hospital of Shandong University approved the present study. Due to the retrospective nature of the study, written informed consent was not available from all participants.

Preoperative preparation. Iodine scrubbing and scanning with iodine contrast materials were prohibited for 1 month. All patients were required to discontinue levothyroxine sodium tablets for at least one month following surgery, until the serum thyrotropin (TSH) concentration was $>30 \mathrm{mIU} / 1$. Patients were maintained on a low iodine diet for 2 weeks prior to the scan. Serum TSH, Tg, anti-thyroglobulin (Anti-Tg), routine blood examination, hepatic and renal function, electrocardiogram, neck ultrasound and chest X-ray were measured $\sim 2-3$ days prior to administration of the diagnostic dose. Patients were hospitalized in the special ward of the Department of Endocrinology, Qilu Hospital of Shandong University (Jinan, Shandong), according to European Union legislations (15). The 24-h ${ }^{131}$ I pre-treatment uptake value in the neck region was measured using a thyroid function machine (MN-6110; Zhongke Zhongjia Scientific Instrument Co., Ltd., Anhui, China). A total of $1 \mathrm{mCi}$ of ${ }^{131} \mathrm{I}$ was administered orally, followed by planar scintigraphy of the neck region $24 \mathrm{~h}$ later. The uptake rate of ${ }^{131} \mathrm{I}$ was used to avoid unnecessary exposure and local radioiodine side-effects (16). Uptake rates $<5 \%$, between $5-10 \%$ and $>10 \%$ was followed by 100,80 or $30 \mathrm{mCi}$ of ${ }^{131} \mathrm{I}$ in one dose for remnant ablation, respectively. Generally, uptake rate was $<5 \%$ following total thyroidectomy. A total of $2 \mathrm{~h}$ later, the patients were encouraged to drink more water, resulting in increased rates of urination. Oral prednisone and vitamin $\mathrm{C}$ were administered to alleviate the local radioiodine side effects. The patients continued receiving levothyroxine sodium tablets replacements $48 \mathrm{~h}$ later, to suppress TSH levels to $<0.1-0.5 \mathrm{mIU} / \mathrm{l}$. A WBIS was performed 5-7 days following ${ }^{131}$ I administration to ensure proper tracer localization in the residual functioning thyroid tissues in the neck, and to confirm the presence of any cervical lymph node or distant functioning metastases. A panel of 3 nuclear medicine physicians performed the aforementioned procedures. The patients with CLNM, as identified by WBIS, received a second ablation with oral $120 \mathrm{mCi}{ }^{131} \mathrm{I} 6$ months following the first ablation. The preparation for the 6-month post-therapy evaluation was similar to that for the pre-ablation scan. If the second ${ }^{131} \mathrm{I}$ treatment failed again, the patients received a third ablation with $150 \mathrm{mCi}{ }^{131} \mathrm{I}$. The decision of repeat ${ }^{131} \mathrm{I}$ administration was based on detectable serum Tg levels or on abnormal uptake in WBIS. Repetitive ${ }^{131} \mathrm{I}$ treatments were administrated until successful ablation was achieved, or until the patient did not tolerate or refuse the treatment, subsequent to which annual check-ups were planned with the measurement of Tg levels.

Outcome assessment. Successful ablation was defined as stimulated serum Tg levels of $<2 \mathrm{ng} / \mathrm{ml}$ with negative Anti-Tg in the absence of cervical lymph node metastases on the ultrasound scan of the neck and on WBIS. Patients who did not fulfill these criteria were considered to exhibit persistent disease. Disease recurrence was monitored in patients who were in remission for $\geq 1$ year, and if a follow-up examination revealed evidence of disease recurrence by imaging and/or raised thyroglobulin levels under either TSH suppression or stimulation. Persistent and recurrent disease was defined as unsuccessful ablation.

Hormone measurements. The ARCHITECT TSH assay was used to measure TSH levels with chemiluminescent microparticle immunoassay (CMIA), with an analytical sensitivity of $0.0025 \mu \mathrm{IU} / 1$ and functional sensitivity of $0.01 \mu \mathrm{IU} / 1$, according to ARCHITECT i system (Abbott Laboratories, Chicago, IL, USA). Tg serum level was measured by an immunochemiluminometric two-site immunoassay, with a calibration range of 0.04-500 ng/ml (Roche Diagnostics GmbH, Mannheim, Germany). The measurement of Anti-Tg was performed 


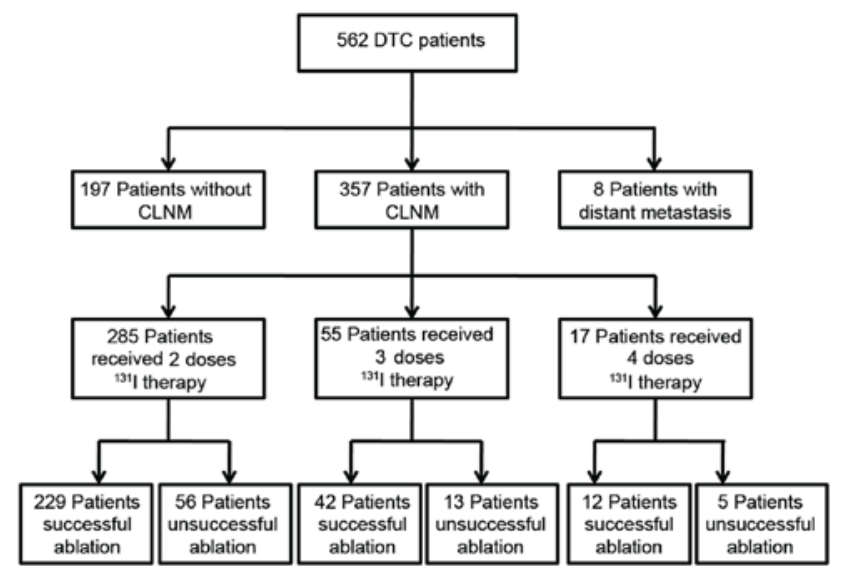

Figure 1. Selection of patients in the present study as shown by flow chart. DTC, differentiated thyroid cancer; CLNM, cervical lymph node metastasis; ${ }^{131}$ I, Radioiodine-131.

using the ARCHITECT Anti-Tg assay with a calibration range of $0.0-1,000.0 \mathrm{IU} / \mathrm{ml}$. The assay was supplied by Fisher Diagnostics (Thermo Fisher Scientific, lnc., Waltham, MA, USA).

Statistical analysis. Statistical analyses were performed with SPSS statistical software (version 16.0; SPSS, Inc., Chicago, IL, USA). Categorical values are presented as relative frequencies. A $\chi^{2}$ test was used to compare categorical data. Univariate Cox proportional hazards regression analysis was used to determine the factors affecting survival time. All possible predictive variables were entered into multivariate logistic model with a backwards stepwise method to remove the least predictive variable at each step. A two-tailed $\mathrm{P}<0.05$ was considered to indicate a statistically significant difference.

\section{Results}

General characteristics of the study population. The present study retrospective study involved 562 patients with DTC who were referred to the Department of Endocrinology, Qilu Hospital of Shandong University (Jinan, Shandong), for ${ }^{131}$ I-postoperative ablation, including 197 patients without CLNM, 357 patients with CLNM and 8 patients with distant metastasis. In general, the patients with DTC with CLNM received remnant ablation and repetitive administration of ${ }^{131} \mathrm{I}$. The duration of ${ }^{131}$ I therapy was based either on detectable serum Tg levels or abnormal absorbance in WBIS. A total of 357 patients with DTC with CLNM received administration of ${ }^{131} \mathrm{I}$, in which 285,55 and 17 patients received 2, 3 or 4 doses of ${ }^{131} \mathrm{I}$ therapy, respectively. The rate of successful ablation was $80.35(229 / 285), 76.36(42 / 55)$ and $70.59(12 / 17)$ in these three groups, respectively. The flowchart for the inclusion of all the participants is described in Fig. 1. The clinical characteristics of the participants are summarized in Table I. The typical WBIS scanning of the process of receiving ${ }^{131} \mathrm{I}$ administration is presented in Figs. 2-4.

Successful ablation was associated with clinical characteristics in patients with DTC with CLNM who received 2 doses of ${ }^{131} I$ therapy. To identify potential effects of the clinical
Table I. Baseline characteristics of the patients with differentiated thyroid cancer with cervical lymph node metastasis.

\begin{tabular}{|c|c|c|c|}
\hline \multirow[b]{2}{*}{ Characteristics } & \multicolumn{3}{|c|}{$\begin{array}{l}\text { Doses of }{ }^{131} \mathrm{I} \\
\text { therapy }(\mathrm{N})\end{array}$} \\
\hline & $2(n=285)$ & $3(n=55)$ & $4(n=17)$ \\
\hline \multicolumn{4}{|l|}{ Age, years } \\
\hline$<45$ & 139 & 28 & 7 \\
\hline$\geq 45$ & 146 & 27 & 10 \\
\hline \multicolumn{4}{|l|}{ Sex } \\
\hline Male & 68 & 20 & 6 \\
\hline Female & 217 & 35 & 11 \\
\hline \multicolumn{4}{|l|}{ Previous thyroid surgery } \\
\hline Near-total thyroidectomy & 19 & 12 & 3 \\
\hline Total thyroidectomy & 266 & 43 & 14 \\
\hline \multicolumn{4}{|l|}{ Histopathological types } \\
\hline Papillary carcinoma & 276 & 53 & 16 \\
\hline Follicular carcinoma & 9 & 2 & 1 \\
\hline \multicolumn{4}{|l|}{ Size of tumor, cm } \\
\hline$<2$ & 171 & 22 & 6 \\
\hline $2-4$ & 91 & 28 & 7 \\
\hline$>4$ & 23 & 5 & 4 \\
\hline \multicolumn{4}{|l|}{ Number of nodules } \\
\hline Solitary & 97 & 16 & 6 \\
\hline Multiple & 188 & 39 & 11 \\
\hline \multicolumn{4}{|l|}{ Capsular invasion } \\
\hline Yes & 259 & 52 & 16 \\
\hline No & 26 & 3 & 1 \\
\hline \multicolumn{4}{|l|}{ TNM stage } \\
\hline Stage I-II & 140 & 27 & 7 \\
\hline Stage III-IV & 145 & 28 & 10 \\
\hline \multicolumn{4}{|l|}{ RRS } \\
\hline Low risk & 0 & 0 & 0 \\
\hline Intermediate risk & 259 & 47 & 9 \\
\hline High risk & 26 & 8 & 8 \\
\hline \multicolumn{4}{|c|}{$\begin{array}{l}\text { Time from surgery to ablation, } \\
\text { month }\end{array}$} \\
\hline$\leq 1$ & 150 & 34 & 5 \\
\hline$>1$ & 135 & 21 & 12 \\
\hline \multicolumn{4}{|l|}{ Ablation } \\
\hline Successful ablation & 229 & 42 & 12 \\
\hline Unsuccessful ablation & 56 & 13 & 5 \\
\hline
\end{tabular}

TNM, tumor node metastasis; RRS, recurrence risk stratification.

features on successful ablation, the differences in clinical pathological characteristics between patients with successful and unsuccessful ablation were compared. Due to the limited data of the patients who received 3 and 4 doses of ${ }^{131}$ I treatment, the analysis of these results was excluded, and the present study focused on the patients who received 2 doses of ${ }^{131} \mathrm{I}$ therapy. The proportion of patients with successful 


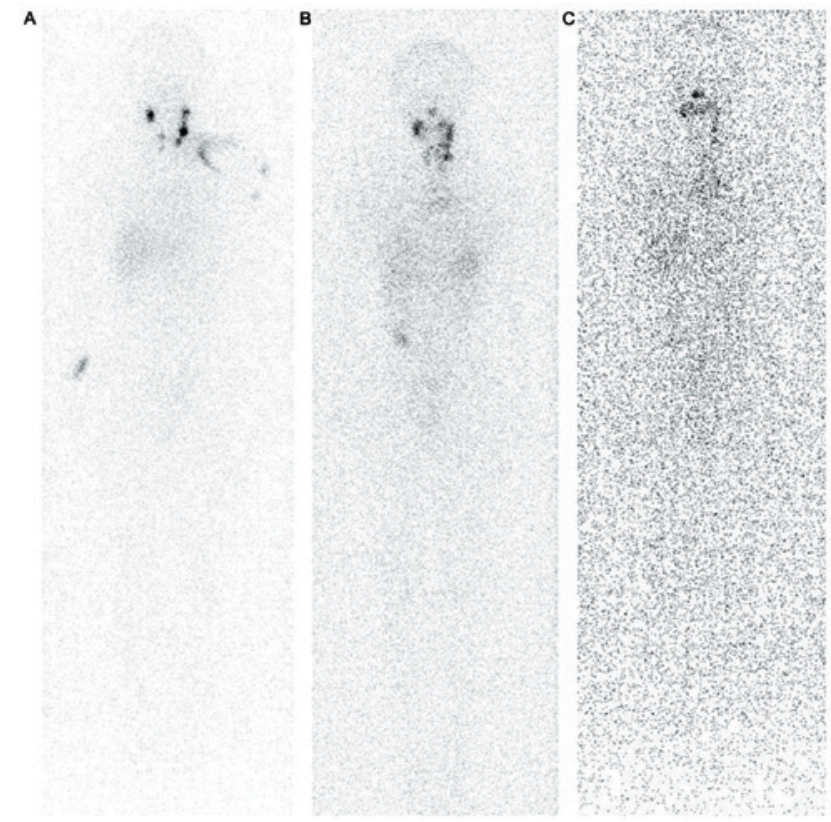

Figure 2. WBIS in a patient with differentiated thyroid cancer and cervical lymph node metastasis who received 2 doses of ${ }^{131} \mathrm{I}$. (A) Postoperative diagnostic WBIS. (B) Follow-up at 6 months subsequent to remnant ablation with $100 \mathrm{mCi}{ }^{131} \mathrm{I}$. (C) Successful ablation was achieved at 6 months following a second dose of $120 \mathrm{mCi}{ }^{131} \mathrm{I}$. WBIS, Whole-body iodine scan; ${ }^{131}$ I, Radioiodine-131.

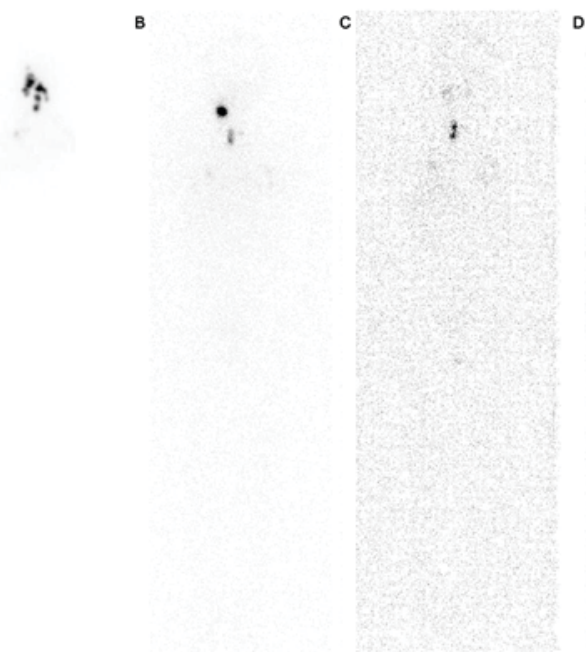

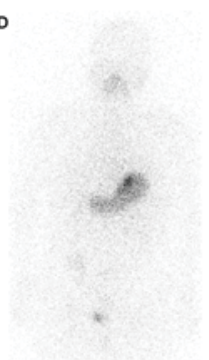

c

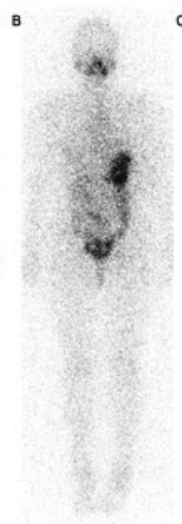

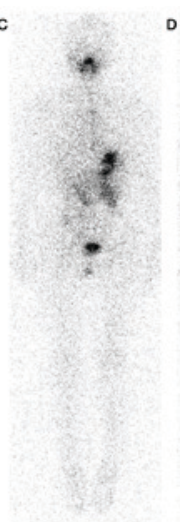

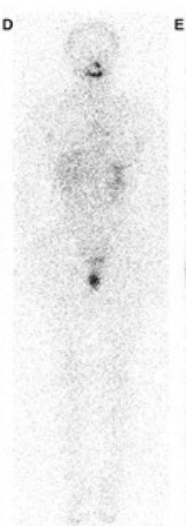

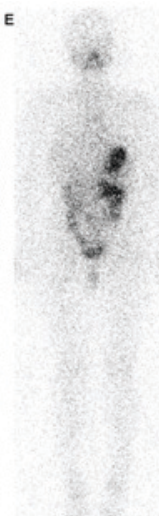

Figure 4. WBIS in a patient with differentiated thyroid cancer with cervical lymph node metastasis who received 4 doses of ${ }^{131} \mathrm{I}$. (A) Postoperative diagnostic WBIS. (B) Follow-up at 6 months following remnant ablation with $100 \mathrm{mCi}{ }^{131} \mathrm{I}$. (C) Follow-up at 6 months following a second dose of $120 \mathrm{mCi}{ }^{131} \mathrm{I}$. (D) Follow-up at 6 months following a third dose of $150 \mathrm{mCi}$ ${ }^{131}$ I. (E) Successful ablation was achieved at 6 months following a fourth dose of $150 \mathrm{mCi}{ }^{131} \mathrm{I}$. WBIS, Whole-body iodine scan; ${ }^{131} \mathrm{I}$, Radioiodine-131.

histopathological type $(\mathrm{P}=0.692)$, capsular invasion $(\mathrm{P}=0.107)$, RSS risk $(\mathrm{P}=0.134)$ and time interval from surgery to ablation $(\mathrm{P}=0.660)$. However, a significantly higher rate of successful ablation was identified in patients who were $<45$ years old compared with patients who were $\geq 45$ years old $(\mathrm{P}<0.001)$. There was also a significantly higher level of successful ablation in patients with tumor size $<2 \mathrm{~cm}$ compared with patients with tumor size $\geq 2 \mathrm{~cm}(\mathrm{P}<0.001)$. Notably, successful ablation was significantly higher in patients with solitary nodules compared with patients with multiple nodules $(\mathrm{P}=0.026)$. Additionally, the patients with stages I-II disease exhibited a higher successful ablation rate compared with patients with stage III-IV disease $(\mathrm{P}<0.001)$. These results indicated that successful ablation may be affected, or partly affected, by certain critical clinical factors, including age, tumor size, number of nodules and TNM stages in patients with DTC with CLNM.

Independent risk factor for successful ablation in DTC patients with CLNM who received two doses of ${ }^{131} I$ therapy. As the $\chi^{2}$ test for the association of categorical variables revealed that age, tumor size, number of nodules and TNM stage are able to affect the rate of successful ablation, these factors may be independent risk factors for successful ablation in patients with DTC with CLNM who received 2 doses of ${ }^{131}$ I therapy.

The present study identified the potential risk factors for successful ablation using univariate and multivariate logistic regression analysis. As indicated in Table III, univariate logistic regression identified no significant association of successful ablation with sex $(\mathrm{P}=0.899)$, type of thyroid surgery $(\mathrm{P}=0.411)$, histopathological types $(\mathrm{P}=0.844)$, capsular invasion ( $\mathrm{P}=0.316)$, RSS risk $(\mathrm{P}=0.512)$ and time interval from surgery to ablation $(\mathrm{P}=0.497)$. Notably, age $(\mathrm{P}=0.003)$, tumor size $(\mathrm{P}<0.001)$, number of nodules $(\mathrm{P}=0.029)$ and $\mathrm{TNM}$ stage $(\mathrm{P}<0.001)$ were significantly associated with successful ablation. Multivariate analysis indicated that age $(\mathrm{P}=0.183)$ was not significantly associated with the rate of successful ablation, but tumor size $(\mathrm{P}<0.001)$, number of nodules $(\mathrm{P}=0.012)$ and TNM stage $(\mathrm{P}<0.001)$ remained significantly associated with the rate to sex $(P=0.899)$, type of thyroid surgery $(P=0.548)$, 
Table II. Comparison of baseline characteristics of successful and unsuccessful ablation in patients with differentiated thyroid cancer with cervical lymph node metastasis who received two doses of radioiodine-131 therapy.

\begin{tabular}{|c|c|c|c|}
\hline Characteristics & Successful ablation, n (\%) & Unsuccessful ablation, n (\%) & P-value \\
\hline \multicolumn{4}{|l|}{ Age, years } \\
\hline$<45$ & $126(90.65)$ & $13(9.35)$ & \multirow[t]{2}{*}{$<0.001$} \\
\hline$\geq 45$ & $103(70.55)$ & $43(29.45)$ & \\
\hline \multicolumn{4}{|l|}{ Sex } \\
\hline Male & $55(80.88)$ & $13(19.12)$ & \multirow[t]{2}{*}{0.899} \\
\hline Female & $174(80.18)$ & $43(19.82)$ & \\
\hline \multicolumn{4}{|l|}{ Previous thyroid surgery } \\
\hline Near-total thyroidectomy & $14(73.68)$ & $5(26.32)$ & \multirow[t]{2}{*}{0.548} \\
\hline Total thyroidectomy & $215(80.83)$ & $51(19.17)$ & \\
\hline \multicolumn{4}{|l|}{ Histopathological type } \\
\hline Papillary carcinoma & $222(80.43)$ & $54(19.57)$ & \multirow[t]{2}{*}{0.692} \\
\hline Follicular carcinoma & $7(77.78)$ & $2(22.22)$ & \\
\hline \multicolumn{4}{|l|}{ Size of tumor, $\mathrm{cm}$} \\
\hline$<2$ & $157(91.81)$ & $14(8.19)$ & \multirow[t]{2}{*}{$<0.001$} \\
\hline$\geq 2$ & $72(63.16)$ & $42(36.84)$ & \\
\hline \multicolumn{4}{|l|}{ Number of nodules } \\
\hline Solitary & $85(87.63)$ & $12(12.37)$ & \multirow[t]{2}{*}{0.026} \\
\hline Multiple & $144(76.60)$ & $44(23.40)$ & \\
\hline \multicolumn{4}{|l|}{ Capsular invasion } \\
\hline Yes & $205(79.15)$ & $54(20.85)$ & \multirow[t]{2}{*}{0.107} \\
\hline No & $24(92.31)$ & $2(7.69)$ & \\
\hline \multicolumn{4}{|l|}{ TNM stage } \\
\hline Stage I-II & $128(91.43)$ & $12(8.57)$ & \multirow[t]{2}{*}{$<0.001$} \\
\hline Stage III-IV & $101(69.66)$ & $44(30.34)$ & \\
\hline \multicolumn{4}{|l|}{ RRS } \\
\hline Intermediate risk & $211(81.47)$ & $48(18.53)$ & \multirow[t]{2}{*}{0.134} \\
\hline High risk & $18(69.23)$ & $8(30.77)$ & \\
\hline \multicolumn{4}{|c|}{ Time from surgery to ablation, month } \\
\hline$\leq 1$ & $122(81.33)$ & $28(18.67)$ & \multirow[t]{2}{*}{0.660} \\
\hline$>1$ & $107(79.26)$ & $28(20.74)$ & \\
\hline
\end{tabular}

TNM, tumor node metastasis; RRS, recurrence risk stratification.

of successful ablation. These results revealed that tumor size, number of nodules and TNM stage may be independent risk factors associated with successful ablation in patients with DTC with CLNM who received 2 doses of ${ }^{131}$ I therapy.

\section{Discussion}

CLNM is common in DTC, with a frequency of 30-90\% (17) and has been demonstrated to be closely associated with higher recurrence and poorer survival rates in patients with DTC (18). The majority of metastatic sites are located in central compartment lymph nodes, and complete central lymph node dissection has been identified to reduce the incidence of disease persistence, recurrence and mortality (19). However, when lymph nodes metastasis is not restricted to the central compartment, post-operative remnant ablation is supplemented to eradicate remnant CLNM. Previous studies have demonstrated that remnant ablation is an independent variable that reduces loco-regional recurrence, distant metastases and cancer-associated mortality (20). However, no single group achieved $100 \%$ ablation following remnant ablation (21). The majority of patients with DTC with CLNM required repetitive ${ }^{131} \mathrm{I}$ treatments $(22)$. Therefore, the present retrospective study of 357 patients with DTC with CLNM was performed to investigate the rate of successful ablation, and to analyze the effects of possible prognostic factors affecting successful ablation, including age, tumor size, number of nodules and TNM stage.

In the present study, although one dose of $100 \mathrm{mCi}{ }^{131} \mathrm{I}$ was administered, successful remnant ablation was not achieved in any of the patients with DTC and CLNM. This is in contrast with a number of studies that have demonstrated that the 
Table III. Univariate and multivariate Cox regression analyses in patients with differentiated thyroid cancer with cervical lymph node metastasis who received two doses of radioiodine-131 therapy $(n=285)$ and the effect of successful ablation.

A, Univariate analysis

\begin{tabular}{lrrr}
\hline Variable & HR & 95\% CI & P-value \\
\hline Age & 0.963 & $0.940-0.987$ & 0.003 \\
Sex & 1.046 & $0.524-2.085$ & 0.899 \\
Type of thyroid surgery & 1.521 & $0.560-4.130$ & 0.411 \\
Histopathological type & 1.175 & $0.237-5.814$ & 0.844 \\
Tumor size & 0.460 & $0.354-0.600$ & $<0.001$ \\
Number of nodules & 2.164 & $1.083-4.325$ & 0.029 \\
Capsular invasion & 0.316 & $0.072-1.380$ & 0.316 \\
TNM stage & 0.243 & $0.124-0.476$ & $<0.001$ \\
ETA risk & 0.512 & $0.210-1.246$ & 0.512 \\
Time from surgery to ablation & 0.817 & $0.455-1.466$ & 0.497 \\
\hline
\end{tabular}

B, Multivariate analysis

\begin{tabular}{lccc}
\hline Variable & HR & $95 \%$ CI & P-value \\
\hline Age & 1.031 & $0.986-1.079$ & 0.183 \\
Sex & - & - & - \\
Type of thyroid surgery & - & - & - \\
Histopathological types & - & - & - \\
Tumor size & 0.434 & $0.323-0.585$ & $<0.001$ \\
Number of nodules & 2.828 & $1.257-6.360$ & 0.012 \\
Capsular invasion & - & - & - \\
TNM stage & 0.243 & $0.116-0.513$ & $<0.001$ \\
RRS risk & - & - & - \\
Time from surgery to ablation & - & - & -
\end{tabular}

Reference category is 0 : Sex: Male, 1 ; female, 0 ; Type of thyroid surgery: Total thyroidectomy, 1; near-total thyroidectomy, 0 ; Histopathological types: Papillary carcinoma, 1; Follicular carcinoma, 0; Number of nodules: Solitary, 1; multiple, 0; Capsular invasion: Yes, 1; no, 0; TNM stage: Stage I-II, 1; Stage III-IV, 0; Time from surgery to ablation: $\leq 1$ month, $0 ;>1$ month, 1 . HR, hazard ratio; CI, confidence interval; TNM, tumor node metastasis; RRS, recurrence risk stratification.

administration of $100 \mathrm{mCi}{ }^{131} \mathrm{I}$ achieved an acceptable ablation rate, from 50 to $100 \%$ (23-25). The difference in ablation outcomes was associated with the clinical characteristics of the participants: The patients recruited in the present study exhibited CLNM, whereas the patients in the previously reported studies did not exhibit extra-thyroidal extension, nor lymph node or distant metastases, suggesting that lymph node or distant metastases may be an independent risk factor affecting successful remnant ablation.

In clinical practice, patients who fail to achieve complete ablation following remnant ablation should receive a second administration of ${ }^{131} \mathrm{I}$ to achieve successful ablation (26). In the present study, all of these patients received $\geq 2$ doses of
${ }^{131}$ I therapy, with a maximum of 4 doses. The rate of successful ablation was $80.35 \%(229 / 285)$ at 2 doses, $76.36 \%(42 / 55)$ at 3 doses, $70.58 \%(12 / 17)$ at 4 doses. The second, third and fourth ${ }^{131}$ I treatments were administered orally, with 120, 150 and $150 \mathrm{mCi}$, respectively. The rate of successful ablation was in agreement with results from a previous study, which stated that $30-50 \mathrm{mCi}, 75 \mathrm{mCi}$ or $100 \mathrm{mCi}$ as a second ${ }^{131} \mathrm{I}$ treatment achieved successful ablation in patients with DTC confined to the thyroid (27). This may be partially explained by the change in size of remnant thyroid tissue, which is expected to become significantly smaller following a high initial dose of ${ }^{131} \mathrm{I}$. A low dosage may have an almost equal ablation outcome compared with high activity in patients with small remnants of thyroid tissue (12). Similar to patients with DTC restricted to the thyroid, whether patients with DTC with CLNM would be able to use a lower dosage as a second dose to achieve a complete ablation outcome should be examined in future studies, to alleviate local radioiodine side-effects.

In the present study, a number of patients that received 4 doses of ${ }^{131}$ I treatment did not achieve successful ablation. These results indicated that the diminution of remnant lymph node tissues following remnant ablation and the different ${ }^{131}$ I activity was not solely associated with ablation outcomes. Therefore, the factors that may contribute to successful ablation in patients with DTC with CLNM were investigated. Due to a large disparity in the numbers of patients receiving 2,3 and 4 doses of ${ }^{131} \mathrm{I}$, the present analysis concentrated on those receiving 2 doses of ${ }^{131} \mathrm{I}$. It was identified that tumor size, number of nodules and TNM stage were the independent risk factors affecting successful ablation in patients with DTC with CLNM who received 2 doses of ${ }^{131}$ I therapy. Additionally, age was significantly associated with complete ablation in the univariate analysis, particularly in patients aged $\leq 45$ years. It has demonstrated that the delay in initial ${ }^{131}$ I therapy following total thyroidectomy in patients with DTC with metastases resulted in poor survival (28). However, in the present study, the timing of the first ${ }^{131}$ I treatment exhibited no effect on the rate of successful ablation, which was consistent with the findings of Tsirona et al (29).

There are several limitations with the present study. Firstly, the assessment time of successful ablation was between 8 and 55 months. Future recurrence was not addressed due to short follow-up periods. Long-term follow-up should be performed in the future studies to examine distant metastasis and recurrence. Secondly, the participants with CLNM were diagnosed at the entry of this trial, and the changes in lymph node and distant metastasis were not observed during follow-up, which may be a factor contributing to unsuccessful ablation, and should be addressed in future follow-up. Additionally, as there was a large variation in the numbers of patients included in the groups, the analysis between the patients with DTC who received 2, 3 and 4 doses of ${ }^{131} \mathrm{I}$ treatment was not relevant; an increased number of patients should have been recruited. Finally, as the trial was only performed in a single center, the Qilu Hospital of Shandong University, all conclusions should be verified in a multi-center, large-scale cohort.

In conclusion, the present study demonstrated that ${ }^{131} \mathrm{I}$ administration is a useful therapy to eradicate remnant thyroid tissue and cervical lymph node metastasis in patients with differentiated thyroid cancer, and that age, tumor size, number of nodules and TNM stage were independent risk 
factors affecting successful ablation in patients with DTC with CLNM. Therefore, ${ }^{131} \mathrm{I}$ treatment is preferentially indicated in patients with DTC with CLNM who are aged $<45$ years, with tumor sizes $<2 \mathrm{~cm}$, solitary nodules and lower TNM stages in order to control and prevent recurrence and/or metastases.

\section{Acknowledgements}

The authors would like to thank Professor Jun Peng, Wen Wang and Jinbo Liu (Qilu Hospital of Shandong University) for their help in revising the manuscript.

\section{Funding}

No funding was received.

\section{Availability of data and materials}

The data of the present study is be available upon request from the corresponding author.

\section{Authors' contributions}

CJC and CYD contributed to the majority of the experiments, data collection, study design and drafted the manuscript. CJC, CYD, JYL and WLX contributed to data analysis. ZSL, WZ, $\mathrm{LC}$ and $\mathrm{KHZ}$ contributed to data and figure collection. CJC and HL conceived the study. HL was responsible for study supervision

\section{Ethics approval and consent to participate}

The Medical Ethical Committee of Qilu Hospital of Shandong University approved the present study. Informed consent was obtained from each patient before entering this experiment.

\section{Consent for publication}

Informed consent was obtained from each patient before entering this experiment.

\section{Competing interests}

The authors declare that they have no competing interests.

\section{References}

1. Jemal A, Bray F, Center MM, Ferlay J, Ward E and Forman D: Global cancer statistics. CA Cancer J Clin 61: 69-90, 2011.

2. Mitchell AL, Gandhi A, Scott-Coombes D and Perros P Management of thyroid cancer: United kingdom national multidisciplinary guidelines. J Laryngol Otol 130: S150-S160, 2016.

3. Shi RL, Qu N, Yang SW, Ma B, Lu ZW, Wen D, Sun GH, Wang Y and Ji QH: Tumor size interpretation for predicting cervical lymph node metastasis using a differentiated thyroid cancer risk model. Onco Targets Ther 9: 5015-5022, 2016.

4. Wang TS, Dubner S, Sznyter LA and Heller KS: Incidence of metastatic well-differentiated thyroid cancer in cervical lymph nodes. Arch Otolaryngol Head Neck Surg 130: 110-113, 2004.

5. Hay ID, Grant CS, van Heerden JA, Goellner JR, Ebersold JR and Bergstralh EJ: Papillary thyroid microcarcinoma: A study of 535 cases observed in a 50-year period. Surgery 112: 1139-1147, 1992.
6. Lang BH, Ng SH, Lau LL, Cowling BJ, Wong KP and Wan KY: A systematic review and meta-analysis of prophylactic central neck dissection on short-term locoregional recurrence in papillary thyroid carcinoma after total thyroidectomy. Thyroid 23: 1087-1098, 2013

7. Hundahl SA, Cady B, Cunningham MP, Mazzaferri E, McKee RF, Rosai J, Shah JP, Fremgen AM, Stewart AK and Hölzer S: Initial results from a prospective cohort study of 5583 cases of thyroid carcinoma treated in the united states during 1996. U.S. and German thyroid cancer study group. An American college of surgeons commission on cancer patient care evaluation study. Cancer 89: 202-217, 2000.

8. Ain KB: Radioiodine-remnant ablation in low-risk differentiated thyroid cancer: Pros Endocrine 50: 61-66, 2015.

9. Schlumberger M, Catargi B, Borget I, Deandreis D, Zerdoud S, Bridji B, Bardet S, Leenhardt L, Bastie D, Schvartz C, et al: Strategies of radioiodine ablation in patients with low-risk thyroid cancer. N Engl J Med 366: 1663-1673, 2012.

10. Lee YM, Cho JY, Sung TY, Kim TY, Chung KW, Hong SJ and Yoon JH: Clinicopathological risk factors and biochemical predictors of safe discharge after total thyroidectomy and central compartment node dissection for thyroid cancer: A prospective study. Int J Endocrinol 2015: 214525, 2015.

11. Jia Q, Meng Z, Tan J,Zhang G, He Y, Sun H, Yu C, Li D, Zheng W, Wang R, et al: Retrospective imaging study on the diagnosis of pathological false positive iodine-131 scans in patients with thyroid cancer. Exp Ther Med 10: 1995-2001, 2015.

12. Hackshaw A, Harmer C, Mallick U, Haq M and Franklyn JA: 131I activity for remnant ablation in patients with differentiated thyroid cancer: A systematic review. J Clin Endocrinol Metab 92: 28-38, 2007.

13. Edge SB and Compton CC: The American Joint Committee on Cancer: The 7th edition of the AJCC cancer staging manual and the future of TNM. Ann Surg Oncol 17: 1471-1474, 2010.

14. American Thyroid Association (ATA) Guidelines Taskforce on Thyroid Nodules and Differentiated Thyroid Cancer, Cooper DS, Doherty GM, Haugen BR, Kloos RT, Lee SL, Mandel SJ, Mazzaferri EL, McIver B, Pacini F, et al: Revised American Thyroid Association management guidelines for patients with thyroid nodules and differentiated thyroid cancer. Thyroid 19: 1167-1214, 2009.

15. Harding LK: Radiation protection legislation. Eur J Nucl Med 25: 187-191, 1998.

16. Alexander C, Bader JB, Schaefer A, Finke C and Kirsch CM: Intermediate and long-term side effects of high-dose radioiodine therapy for thyroid carcinoma. J Nucl Med 39: 1551-1554, 1998.

17. Chang H, Yoo RN, Kim SM, Kim BW, Lee YS, Lee SC, Chang HS and Park CS: The clinical significance of the right para-oesophageal lymph nodes in papillary thyroid cancer. Yonsei Med J 56: 1632-1637, 2015.

18. Gulcelik MA, Ozdemir Y, Kadri Colakoglu M, Camlibel M and Alagol $\mathrm{H}$ : Prognostic factors determining survival in patients with node positive differentiated thyroid cancer: A retrospective cross-sectional study. Clin Otolaryngol 37: 460-467, 2012.

19. American Thyroid Association Surgery Working Group; American Association of Endocrine Surgeons, American Academy of Otolaryngology-Head and Neck Surgery, American Head and Neck Society, Carty SE, Cooper DS, Doherty GM, Duh QY, Kloos RT, Mandel SJ, et al: Consensus statement on the terminology and classification of central neck dissection for thyroid cancer. Thyroid 19: 1153-1158, 2009.

20. Bal CS, Kumar A and Pant GS: Radioiodine dose for remnant ablation in differentiated thyroid carcinoma: A randomized clinical trial in 509 patients. J Clin Endocrinol Metab 89: 1666-1673, 2004.

21. M El-Refaei S, W Yassin S, Salman K, Al Munshy T, Al-Ezzi M, M Al-Sayed Y and Abd Elkareem Husseni M: Comparison between low and high radioactive iodine (131I) reablation dose in patients with papillary thyroid cancer. Nucl Med Commun 36: 114-119, 2015.

22. Lim YC, Liu L, Chang JW and Koo BS: Lateral lymph node recurrence after total thyroidectomy and central neck dissection in patients with papillary thyroid cancer without clinical evidence of lateral neck metastasis. Oral Oncol 62: 109-113, 2016.

23. Wagieh SM, El-Refaei SM, Salem SS, Al-Shiekh EA, Al-Ghamdy HA and Al-Juhani NR: Impact of histopathology of non-neoplastic thyroid tissue on ablation outcome in patients with papillary thyroid cancer. Nucl Med Commun 32: 597-604, 2011. 
24. Pacini F, Schlumberger M, Harmer C, Berg GG, Cohen O, Duntas L, Jamar F, Jarzab B, Limbert E, Lind P, et al: Post-surgical use of radioiodine (131I) in patients with papillary and follicular thyroid cancer and the issue of remnant ablation: A consensus report. Eur J Endocrinol 153: 651-659, 2005.

25. Tsang RW, Brierley JD, Simpson WJ, Panzarella T, Gospodarowicz MK and Sutcliffe SB: The effects of surgery, radioiodine, and external radiation therapy on the clinica outcome of patients with differentiated thyroid carcinoma. Cancer 82: 375-388, 1998.

26. McCowen KD, Adler RA, Ghaed N, Verdon T and Hofeldt FD: Low dose radioiodide thyroid ablation in postsurgical patients with thyroid cancer. Am J Med 61: 52-58, 1976.

27. Prpic M, Dabelic N, Stanicic J, Jukic T, Milosevic M and Kusic Z: Adjuvant thyroid remnant ablation in patients with differentiated thyroid carcinoma confined to the thyroid: A comparison of ablation success with different activities of radioiodine (I-131). Ann Nucl Med 26: 744-751, 2012.
28. Higashi T, Nishii R, Yamada S, Nakamoto Y, Ishizu K, Kawase S, Togashi K, Itasaka S, Hiraoka M, Misaki T and Konishi J: Delayed initial radioactive iodine therapy resulted in poor survival in patients with metastatic differentiated thyroid carcinoma: A retrospective statistical analysis of 198 cases. J Nucl Med 52: 683-689, 2012.

29. Tsirona S, Vlassopoulou V, Tzanela M, Rondogianni P, Ioannidis G, Vassilopoulos C, Botoula E, Trivizas P, Datseris I and Tsagarakis S: Impact of early vs late postoperative radioiodine remnant ablation on final outcome in patients with low-risk well-differentiated thyroid cancer. Clin Endocrinol (Oxf) 80: 459-463, 2014. 